\title{
Phospholipid Metabolism in Stimulated Human Platelets
}

\author{
CHANGES IN PHOSPHATIDYLINOSITOL, PHOSPHATIDIC ACID,
} AND LYSOPHOSPHOLIPIDS

\author{
M. Johan Broekman, Jean W. Ward, and Aaron J. Marcus, Divisions of Hematology- \\ Oncology, Departments of Medicine, New York Veterans Administration Hospital, \\ New York, New York 10010; Cornell University Medical College, New York, \\ New York 10021
}

A в S T R A C T Endogenous phospholipid metabolism in stimulated human platelets was studied by phosphorus assay of major and minor components following separation by two-dimensional thin-layer chromatography. This procedure obviated the use of radioactive labels. Extensive changes were found in quantities of phosphatidylinositol (PI) and phosphatidic acid (PA) as a consequence of thrombin or collagen stimulation. Thrombin addition was followed by rapid alterations in the amount of endogenous PI and PA. The decrease in PI was not precisely reciprocated by an increase in PA when thrombin was the stimulus. This apparent discrepancy could be explained by removal of a transient intermediate in PI metabolism, such as diglyceride, formed by PI-specific phospholipase C (Rittenhouse-Simmons, S., J. Clin. Invest. 63: 580-587, 1979). Diglyceride would be unavailable for PA formation by diglyceride kinase, if hydrolyzed by diglyceride lipase (Bell, R. L., D. A. Kennerly, N. Stanford, and P. W. Majerus. Proc. Natl. Acad. Sci. U. S. A. 76: 3238-3241, 1979) to yield arachidonate for prostaglandin endoperoxide formation. Thrombintreated platelets also accumulated lysophosphoglycerides. Specifically, lysophosphatidyl ethanolamines accumulated within $15 \mathrm{~s}$ following thrombin addition. Fatty acid and aldehyde analysis indicated phospholipase $\mathbf{A}_{2}$ activity, with an apparent preference for diacyl ethanolamine phosphoglycerides. In the case of collagen, these changes occurred concomitantly with aggregation and consumption of oxygen for prostaglandin endoperoxide formation.

Part of this work was presented at the Annual Meeting of the American Society for Clinical Investigation, Washington, D. C., May 1979 (Clin. Res. 27: 459A, Abstr.).

Dr. Broekman was the recipient of a New York Heart Association Fellowship during these studies, and is at present a Senior Investigator of the New York Heart Association.

Received for publication 15 February 1980 and in revised form 21 April 1980.
These studies of endogenous phospholipid metabolism provide information supporting the existence of two previously postulated pathways for liberation of arachidonic acid from platelet phospholipids: $(a)$ the combined action of PI-specific phospholipase C plus diglyceride lipase yielding arachidonate derived from PI; and $(b)$ a phospholipase $\mathrm{A}_{2}$ acting primarily on diacyl ethanolamine phosphoglyceride.

\section{INTRODUCTION}

Interest in the effects of stimulation upon platelet lipid metabolism focused initially on inositol-containing phospholipids. As in many secretory tissues (1), platelet stimulation increases the turnover of phosphatidylinositol (PI $)^{1}$ and related phospholipids, such as phosphatidic acid (PA) and di- and triphosphoinositide $(2-4)$. The metabolism of these phospholipids is also relatively active in unstimulated platelets $(5,6)$. The turnover of ethanolaminephosphoglycerides (PE), phosphatidylcholine (PC), and sphingomyelin was reported to be very low in both stimulated and unstimulated platelets $(5,6)$. However, platelet stimulation increases de novo synthesis of phosphatidyl serine (7) and PI (8).

Recently the important role of arachidonic acid and its oxygenation products in platelet function has initiated new interest in platelet lipid metabolism (for review, 9). In platelets, arachidonic acid is esterified to the 2-position of phospholipids (10-12). Investigations on the mechanism by which arachidonate is made available to cyclooxygenase and lipoxygenase have initially centered on a putative phospholipase $A_{2}(13-17)$.

\footnotetext{
${ }^{1}$ Abbreviations used in this paper: DPG, diphosphatidylglycerol, cardiolipin; LPC, lysophosphatidylcholine; LPE, lysophosphatidyl ethanolamine; PA, phosphatidic acid; PC, phosphatidylcholine, lecithin; $\mathrm{PE}$, ethanolamine phosphoglycerides; PI, phosphatidylinositol; TLC, thin-layer chromatography.
} 
A relationship between two aspects of lipid metabolism in stimulated platelets, i.e., PI turnover and arachidonate liberation, was recently postulated by Bell et al. (18), and is based upon their demonstration of a diglyceride lipase in platelets. This enzyme would release arachidonate from diglyceride produced by the PI-specific phospholipase C identified by RittenhouseSimmons (19).

In the present study we provide evidence for two pathways for hydrolysis of the 2-position fatty acid from platelet phospholipids: one involves a phospholipase $A_{2}$ of considerable activity, with diacyl ethanolamine phosphoglycerides as primary substrates; the other involves operation of the aforementioned pathway of PI-specific phospholipase C plus diglyceride lipase. In addition, we have shown that upon collagen stimulation PI-PA metabolism, arachidonate oxygenation, and aggregation follow similar time-courses.

\section{METHODS}

Platelet collection and processing. For each experiment $2 \mathrm{U}$ of whole blood were drawn into a plastic pack system (Fenwal 4R1718, Fenwal Laboratories, Div. Travenol Laboratories, Inc., Deerfield, Ill.), and washed platelets were prepared as previously described (20). The blood donors denied having taken medications during the preceding $2 \mathrm{wk}$. Nevertheless, platelet cyclooxygenase activity was checked before platelet processing by stimulating samples of platelet-rich plasma with collagen and measuring $\mathrm{O}_{2}$ consumption as previously described for washed platelets $(20)$. After removal of platelet-poor plasma, the $2 \mathrm{U}$ were combined, washed twice in Tris-citrate $(20)$, resuspended in $0.15 \mathrm{M} \mathrm{KCl}$, and adjusted to a concentration of $10^{10}$ platelets $/ \mathrm{ml}(21)$.

Experimental design. Incubations $\left(37^{\circ} \mathrm{C}\right)$ for lipid studies were carried out in a $2-\mathrm{ml}$ total volume in open tubes, shaken at 120 excursions/min in a Braun shaker equipped with Thermomix 1460 BKU circulator (B. Braun Instruments, San Francisco, Calif.). $\mathrm{O}_{2}$ consumption and aggregation studies were performed using smaller volumes (20) containing platelets and buffer components in the same proportions as in the lipid studies. Time-course experiments, including controls, were also done in open tubes in a constant temperature bath with magnetic stirring at $\sim 1,000 \mathrm{rpm}$. Platelets, $0.5 \mathrm{ml}\left(5 \times 10^{9}\right)$, were added to $1.5 \mathrm{ml}$ of a solution containing $75 \mathrm{mM}$ Tris, $100 \mathrm{mM} \mathrm{KCl,} 5 \mathrm{mM}$ glucose, and $0.35 \%$ defatted bovine serum albumin at $\mathrm{pH}$ 7.4. Where indicated, EGTA ( $5 \mathrm{mM}$, neutralized to $\mathrm{pH} 7.4$ with $\mathrm{KOH}$ ) was also included. Inhibitors (20) (Table III) were added during a 5-min preincubation, after which the appropriate stimulus was introduced. Incubations were continued as required and then stopped by addition of $7 \mathrm{ml}$ chloroform/ methanol 2:5, vol/vol (12).

Lipid extraction and thin-layer chromatography (TLC). Lipids were extracted as previously described $(5,11,12)$. Aggregation did not interfere with this procedure. KCl-EDFA (5) was not used, to avoid interference during spotting of the lipid extract. TLC plates were prepared by slurrying $35 \mathrm{~g}$ silica gel $\mathrm{H}$ in $85 \mathrm{ml} 2.5 \%$ magnesium acetate, and spreading $0.50-\mathrm{mm}$ layers onto acid-cleaned $20 \times 20-\mathrm{cm}$ glass plates using a Quickfit apparatus (Corning Glass Works, Science Products Div., Corning, N. Y.). $(12,22)$. After air drying for at least $24 \mathrm{~h}$, the plates were stored in a desiccantcontaining cabinet. Plates were "activated" for at least $1 \mathrm{~h}$ in a flow of nitrogen kept at $55 \%$ relative humidity (22). Lipid extracts were spotted in this same atmosphere. Between first (chloroform/methanol/ammonia, 65:35:5.5, vol/vol) and second (chloroform/acetone/methanol/acetic acid/water, 3:4:1:1:0.5, vol/vol) dimensional developments $(12,22)$, plates were dried for $1 \mathrm{~h}$ in a stream of dry nitrogen. With this TLC system, separation of major phospholipids, including PI and phosphatidyl serine, as well as such minor components as PA, diphosphatidylglycerol (DPG), lysophosphatidyl ethanolamine (LPE), and lysophosphatidylcholine (LPC), was readily and reproducibly accomplished (Fig. 1) $(12,22)$.

Phosphorus analysis. After two-dimensional TLC, plates were air dried and stained in iodine vapor. Areas containing phosphorus (detected in separate experiments with a modified Dittmer-Lester spray reagent [23]) were scraped and phosphorus content was determined (24). PI, LPE, PA, and DPG were quantitated with high precision, as evidenced by small standard deviations (Table II). Measurements of the phosphorus content of other phospholipids showed larger standard deviations, contributing to the lack of significance in changes observed after stimulation (Fig. 2) (Table II).

Gas-liquid chromatography. TLC plates with samples for fatty acid and aldehyde analysis were kept in an atmosphere of dry nitrogen after 2-dimensional TLC until dried. Areas to be analyzed were identified, scraped, methylated $\left(\mathrm{BF}_{3}\right)$ methanol), and analyzed as previously described $(11,12,25)$. Results were expressed as weight percent of methylated components.

Chemicals. Reagents used were obtained as previously described (20). In addition, albumin was from Sigma Chemical Co., St. Louis, Mo. (A6003); silica gel H, type 60 (Merck \& Co., Rahway, N. J.), from Brinkmann Instruments, Inc., Westbury, N. Y.; solvents were pesticide grade (Nanograde) from Mallinckrodt, Inc., St. Louis, Mo. TLC and gas chromatography supplies were from Supelco, Inc., Bellefonte, Pa. Human $\alpha$-thrombin $(2,300 \mathrm{U} / \mathrm{mg}$ protein) was a gift from Dr. John Fenton II. Collagen was from Hormon-Chemie, Munich, West Germany.

Statistical analysis was performed on a Hewlett-Packard 97 calculator (Hewlett-Packard, Palo Alto, Calif.) using a program to determine the $t$ statistic for two means.

\section{RESULTS}

Effects of thrombin stimulation on the phospholipid composition of washed human platelets. After the addition of thrombin to a washed platelet suspension, two major differences between phospholipids in the control and stimulated samples were discernible (Fig. 1): First, the quantity of platelet PI decreased, whereas the amount of PA increased dramatically. Second, an additional phospholipid was evident (designated LPE in Fig. 1, located between PI and sphingomyelin). The latter was identified as lysophosphatidyl ethanolamine since it was ninhydrin-positive, contained phosphorus, and co-chromatographed with authentic LPE. In addition, gas chromatographic analysis of methylated samples indicated the presence of a large proportion of dimethylacetal derivatives (Table I). In human platelets measurable amounts of plasmalogens (as dimethylacetals) are found only in the ethanolamine phosphoglyceride class (10-12). The absence of polyunsaturated fatty acids in these samples 

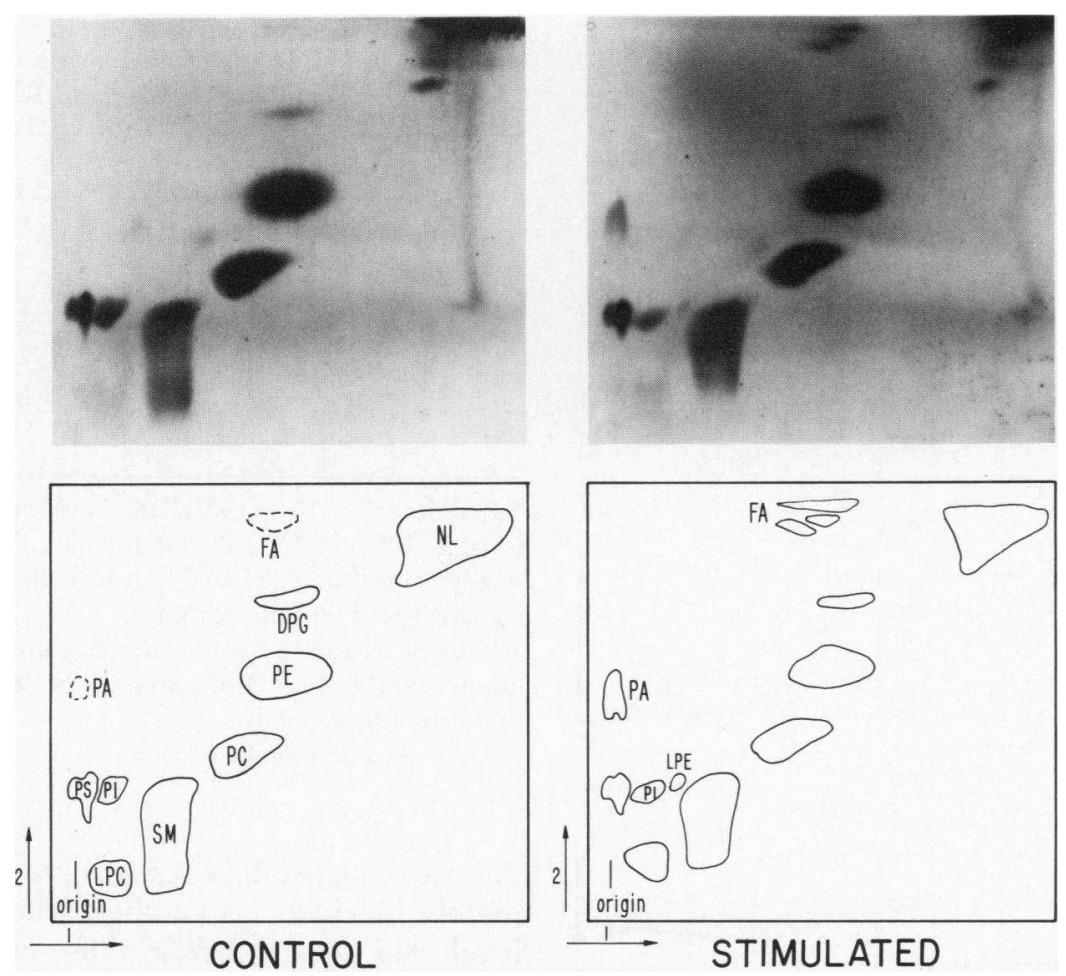

FIGURE 1 Comparison of lipid distribution by two-dimensional TLC of control and thrombin-stimulated platelets. Photograph (top) and tracing (bottom) of a heavily iodine-stained TLC plate of a control incubation $\left(5 \times 10^{9}\right.$ platelets) depict the base-line lipid profile (left). Only trace amounts of PA and fatty acids (FA) are discernible (broken lines); no LPE is visible. Stimulation with thrombin $\left(0.6 \mathrm{U} / 10^{8}\right.$ platelets) for $3 \mathrm{~min}$ (right) altered the lipid pattern: The quantity of PI decreased, PA increased, and a new spot, LPE (absent in the control), appeared. Three distinct spots are seen in the fatty acid area (FA) of the stimulated sample. These were tentatively identified as the hydroxy acids 12-hydroxyeicosatetraenoic acid (HETE) and 12-hydroxyheptadecatrienoic acid (HHT), and free fatty acids. (Thromboxanes are not extracted by the procedure used.) All three zones were radioactive when the platelets were prelabeled with $\left[{ }^{14} \mathrm{C}\right]$ arachidonic acid (data not shown). The line above the word "origin" indicates the position of application of the lipid extract. NL, neutral lipids; for other abbreviations, see text.

(Table I) suggested that the LPE was formed by a phospholipase $\mathbf{A}_{2}$-like activity.

The quantitative changes in platelet phospholipids due to thrombin stimulation are listed in Table II, while their time-courses are depicted by Fig. 2. The changes in PI and PA are rapid $(50 \%$ complete in $20 \mathrm{~s})$, large, and statistically significant $(P<0.001$, Table II). However, it should be noted that the decreases in PI $(17.8 \mathrm{nmol}$ at $60 \mathrm{~s})$ were greater than the increases in PA (10 $\mathrm{nmol}$ at $60 \mathrm{~s})$. The phosphorus content of the LPE area (Fig. 1) rose rapidly from barely detectable levels to a plateau value of $5 \mathrm{nmol}$, a statistically significant change (Fig. 2 and Table II). The level of LPC rose more slowly after thrombin treatment, and the increase was of less statistical significance (Fig. 2 and Table II). Downward changes of variable magnitude were observed in the quantities of $\mathrm{PE}$ and $\mathrm{PC}$, but were not statistically significant. The time-course of changes in PE and LPE differed from those in PC and LPC. The ethanolamine phospholipids rapidly attained plateau levels, but PC and LPC changed at a later time and more slowly (Fig. 2). These trends were observed in every experiment, despite variability in magnitude of the alterations in PE and PC. There were no changes in level of DPG (Fig. 2). Alterations in phosphatidylserine and sphingomyelin content did not follow an identifiable pattern.

Effect of inhibitors of platelet function on PI-PA metabolism. Treatment of $3.7 \times 10^{9}$ platelets with thrombin $\left(0.14 \mathrm{U} / 10^{8}\right.$ platelets) for $10 \mathrm{~min}$ induced a loss of $>40 \%$ of platelet PI and a 17 -fold gain in the quantity of PA (Table III).

5 min before thrombin addition in an experiment as described above, inhibitors of platelet function were preincubated with the platelet suspension. Arachidonic acid oxygenation was blocked with $5,8,11,14$ eicosatetraynoic acid $(30 \mu \mathrm{M})$ - an inhibitor of both 


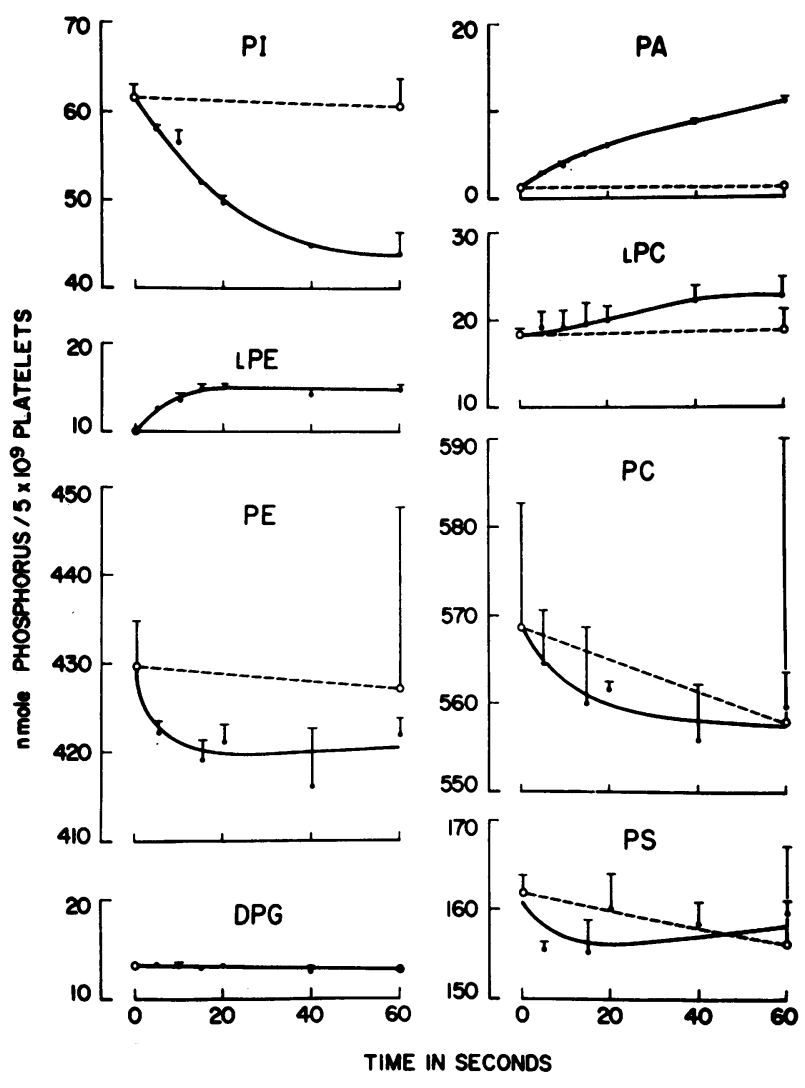

FIGURE 2 Composite representation of quantitative changes in platelet phospholipids following stimulation with thrombin. Washed platelets $\left(5 \times 10^{9}\right)$ were preincubated for $4 \mathrm{~min}$, then stimulated with thrombin $\left(0.2 \mathrm{U} / 10^{8}\right.$ platelets $)$. Incubations were stopped at the indicated time points; lipids were extracted, separated (see Fig. 1), and phosphorus was quantitated as described in Methods. Of note are the increases in PA, LPE, and LPC, as well as the pronounced decrease in PI, and the slight decrease in PE. Equal distances on the ordinate represent identical changes in phosphorus content. Vertical lines indicate SD, which at some points (especially with DPG and PA) were too small to be shown in this figure. $\bigcirc$-.-- $\bigcirc$ control samples; _- stimulated samples. For abbreviations, see text.

the lipoxygenase and cyclooxygenase enzymes (26). Absence of arachidonic acid oxygenation was verified in an oxygen consumption apparatus (20). Indomethacin (Sigma Chemical Co.) $(10,50$, or $100 \mu \mathrm{M})$, a cyclooxygenase inhibitor, was also used. As shown in Table III, preincubation with either inhibitor had no influence on PI-PA metabolism. Imidazole (Sigma Chemical Co.) ( $5 \mathrm{mM})$, a thromboxane synthetase inhibitor, was also without effect on this pathway. In additional experiments, aspirin, whether added in vitro in concentrations up to $200 \mu \mathrm{M}$ or ingested by the blood donor before collection, had no effect on PI-PA metabolism after thrombin stimulation.

When platelet cyclic AMP levels were elevated by preincubation with dibutyryl cyclic AMP ( $3 \mathrm{mM}$ ),
TABLE I

Fatty Acid and Aldehyde Composition of LPE from Thrombin-stimulated Platelets*

\begin{tabular}{lc}
\hline Component & Percent of Total $\$$ \\
\hline 16:0 DMA & 5 \\
$16: 0$ & 8 \\
18:0 DMA & 18 \\
$18: 1$ DMA & 4 \\
$18: 0$ & 56 \\
$18: 1$ & 10 \\
$20: 4$ & Trace \\
\hline
\end{tabular}

* Platelets $\left(7 \times 10^{9}\right.$ cells $\left./ 2.2 \mathrm{ml}\right)$ were incubated with thrombin $\left(0.14 \mathrm{U} / 10^{8}\right.$ platelets) for $60 \mathrm{~s}$. The LPE of four incubations was isolated, pooled, and transesterified before quantitation by gas-liquid chromatography.

\$ Fatty acid methyl esters are designated by number of $\mathrm{C}$ atoms: double bonds of parent fatty acids. DMA, fatty aldehyde dimethylacetals.

$\S$ Expressed as weight percent.

the usual aggregation response to thrombin was completely blocked. Under these conditions PI and PA levels did not differ from those of unstimulated controls (Table III).

The influence of ionized calcium was studied by addition or removal of extracellular calcium as well as by inhibition of intracellular calcium translocation with 8 -( $N, N$-diethylamino)-octyl-3,4,5-trimethoxybenzoate hydrochloride (TMB-8). The latter compound slightly inhibited metabolism of PI and PA (Table III). This inhibition could be reversed by addition of extracellular calcium. Addition of extracellular calcium per se did not affect metabolism of PI and PA but removal of extracellular calcium by EGTA $(5 \mathrm{mM})$ resulted in strong inhibition (Table III). This effect of EGTA was not observed when incubations were terminated within 3 min after thrombin addition. This phenomenon was explored in greater detail with a time-course study (Fig. 3): metabolism of PI and PA was unaffected by the presence of EGTA up to 2 min following thrombin addition. In the absence of EGTA, maximal changes in PI and PA content were attained at $10 \mathrm{~min}$ (Fig. 3). However, in the presence of EGTA values close to those seen in unstimulated controls were obtained at the 30-min time point (Fig. 3). Thus EGTA did not influence the initial phase of PI-PA metabolism following thrombin stimulation, but did exert an effect later in the time-course.

In separate experiments, ionophore A23187 $(1 \mu \mathrm{M}$, $3 \mathrm{~min}$ ) induced only a $19 \%$ decrease in the level of $\mathrm{PI}$, whereas thrombin $\left(0.6 \mathrm{U} / 10^{8}\right.$ platelets, $\left.3 \mathrm{~min}\right)$ reduced the level of PI by $40 \%$. The increase in PA after ionophore addition (to $300 \%$ of unstimulated control) was minor when compared with that induced by throm- 
TABLE II

Phospholipid Content of Control and Thrombin-stimulated Platelets*

\begin{tabular}{lrlcccr}
\hline $\begin{array}{c}\text { Phospholipid } \\
\text { class }\end{array}$ & \multicolumn{2}{c}{ Control } & \multicolumn{5}{c}{ Stimulated } \\
\hline & & & \multicolumn{2}{c}{$15 s$} & \multicolumn{2}{c}{$60 s$} \\
PA & $1.25 \ddagger$ & $(0.06) \S$ & $5.05^{\prime \prime}$ & $(0.20)$ & $11.11^{\prime \prime}$ & $(0.56)$ \\
PI & 61.55 & $(1.51)$ & $51.96^{\prime \prime}$ & $(0.19)$ & $43.73^{\prime \prime}$ & $(2.46)$ \\
LPC & 18.25 & $(0.68)$ & 19.45 & $(2.41)$ & 22.64 ฯ & $(2.08)$ \\
PC & 568.65 & $(14.10)$ & 559.96 & $(8.65)$ & 559.74 & $(3.88)$ \\
LPE & 0.23 & $(0.13)$ & $5.01^{* *}$ & $(0.61)$ & $4.81^{* *}$ & $(0.39)$ \\
PE & 429.49 & $(5.25)$ & $418.98 \uparrow$ & $(2.23)$ & 421.77 & $(1.93)$ \\
PS & 161.92 & $(1.95)$ & 155.28 & $(3.89)$ & 160.37 & $(1.27)$ \\
DPG & 12.57 & $(0.14)$ & 12.21 & $(0.07)$ & 11.89 & $(0.31)$ \\
SM & 220.61 & $(12.66)$ & 203.94 & $(19.45)$ & 219.93 & $(12.77)$
\end{tabular}

* Platelets $\left(5 \times 10^{9}\right.$ cells $\left./ 2 \mathrm{ml}\right)$ were preincubated for $4 \mathrm{~min}$. Incubations were stopped at 15 and $60 \mathrm{~s}$ after stimulation $\left(0.2 \mathrm{U}\right.$ thrombin $/ 10^{8}$ platelets $)$. Controls were stopped after preincubation. Phospholipid phosphorus in each phospholipid class was determined as described in Methods. PS, phosphatidyl serine; SM, sphingomyelin.

Mean of three incubations is expressed as nanomoles phosphorus per $5 \times 10^{9}$ platelets.

$\S$ SD of three incubations.

" Compared with control, $P<0.001$.

I Compared with control, $P<0.05$.

** Compared with control, $P<0.01$.

bin (1,300\% of control). This increase in PA following ionophore addition was less than the decrease in PI.

Collagen-induced PI-PA metabolism. Platelet content of PI and PA was measured following 3-min incubations with varying amounts of collagen. As in the case of thrombin stimulation, levels of PI and PA changed markedly (Fig. 4). These changes were readily observed, even when the amount of collagen added was $<10 \mu \mathrm{g} / 5 \times 10^{9}$ platelets (Fig. 4), an amount comparable to $0.3 \mu \mathrm{g}$ collagen added to $0.5 \mathrm{ml}$ platelet-

TABLE III

Effects of Inhibitors on Changes in PA and PI Content of Platelets Following Thrombin Stimulation*

\begin{tabular}{lrrrr}
\hline \multicolumn{1}{c}{ Inhibitor } & \multicolumn{2}{c}{ PA } & \multicolumn{2}{c}{ PI } \\
\hline Unstimulated control & $1.30 \neq(100) \S$ & $44.91(100)$ \\
Stimulated control & $22.21(1,700)$ & $25.71(57)$ \\
ETYA $(30 \mu \mathrm{M})$ & $20.67(1,600)$ & $22.97(51)$ \\
Indomethacin $(100 \mu \mathrm{M})$ & $20.23(1,550)$ & $26.16(58)$ \\
Imidazole $(5 \mathrm{mM})$ & $24.70(1,900)$ & 23.99 & $(53)$ \\
Dibutyryl cyclic AMP $(3 \mathrm{mM})$ & $1.50(115)$ & $42.33(94)$ \\
CaCl $(2 \mathrm{mM})$ & $20.83(1,600)$ & $22.59(50)$ \\
EGTA $(5 \mathrm{mM})$ & $5.02(385)$ & $38.77(86)$ \\
TMB-8 $(600 \mu \mathrm{M})$ & $16.07(1,200)$ & 28.42 & $(63)$ \\
TMB-8 $(600 \mu \mathrm{M})+\mathrm{CaCl}_{2}(2 \mathrm{mM})$ & $23.00(1,750)$ & $20.55(46)$ \\
\hline
\end{tabular}

* Platelets $\left(3.7 \times 10^{9}\right.$ cells $\left./ 2 \mathrm{ml}\right)$ were preincubated $(5 \mathrm{~min})$ with buffer or inhibitor(s) at the final concentrations indicated, prior to stimulation with thrombin $\left(0.14 \mathrm{U} / 10^{8}\right.$ platelets) for $10 \mathrm{~min}$. Incubations were stopped, and amounts of PA and PI quantitated as described in Methods.

\$ Mean of duplicate experiments, expressed in nanomoles of phosphorus per 3.7 $\times 10^{9}$ platelets.

$\$$ Percent of unstimulated control.

ETYA, 5,8,11,14-eicosatetraynoic acid; TMB-8, 8-( $N, N$-diethylamino)-octyl-3, 4,5-trimethoxybenzoate hydrochloride. 


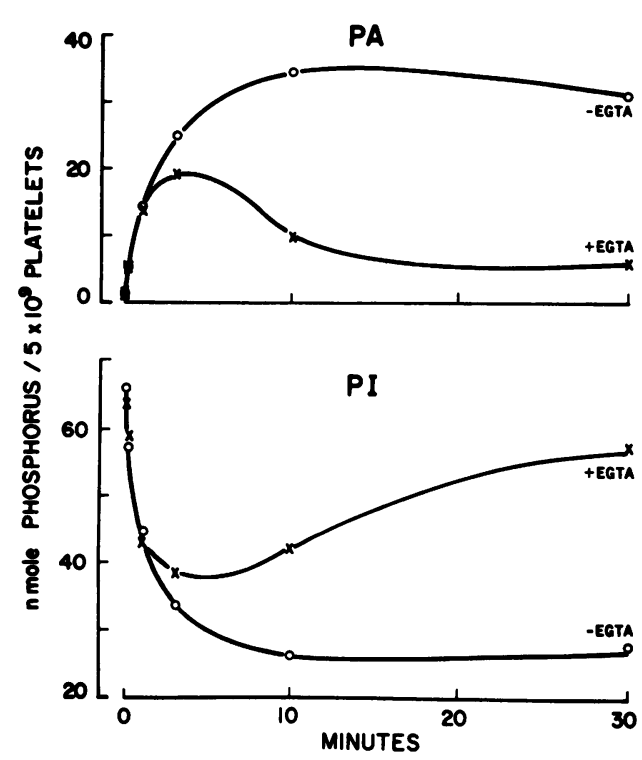

Figure 3 Effect of EGTA on the time-course of changes in quantity of PA (top) and PI (bottom). In the absence of EGTA (O, -EGTA) the changes were initially very rapid, then slowly leveled off. In the presence of EGTA ( $\times,+$ EGTA) the changes were initially equally rapid, but leveled off sooner, and then reversed to approach base-line values between 10 and $30 \mathrm{~min}$ after addition of stimulus $\left(5 \times 10^{9}\right.$ platelets, $0.2 \mathrm{U}$ thrombin $/ 10^{8}$ cells).

rich plasma containing 300,000 platelets/ $\mu$ l. Although accumulation of LPE was observed following collagen stimulation, the primary emphasis of experiments with this stimulus was on the time-course of changes in PI and PA content.

A representative time-course of PI-PA metabolism following collagen stimulation is shown in Fig. 5, together with an aggregation curve and an oxygen consumption trace. Following a lag phase of about $20 \mathrm{~s}$ the rate of oxygen consumption increased in a burst (prostaglandin endoperoxide formation), returning to base-line values $\sim 1 \mathrm{~min}$ later (Fig. 5, [20]). Aggregation, as well as changes in PI and PA content, followed a time-course very similar to that of the burst (Fig. 5). However, whereas PI levels remained at base line before decreasing, PA levels consistently decreased slightly before increasing. Thereafter the gain in endogenous PA equalled the loss in PI (Fig. 5). This was in contrast to the data obtained after thrombin stimulation, where the loss in PI was greater than the gain in PA.

\section{DISCUSSION}

Thrombin-induced changes in endogenous platelet phospholipids. Thrombin stimulation of platelets induced major changes in endogenous PI and PA content (Fig. 1). A discrepancy was observed between loss of PI and gain in PA: the loss of PI was greater

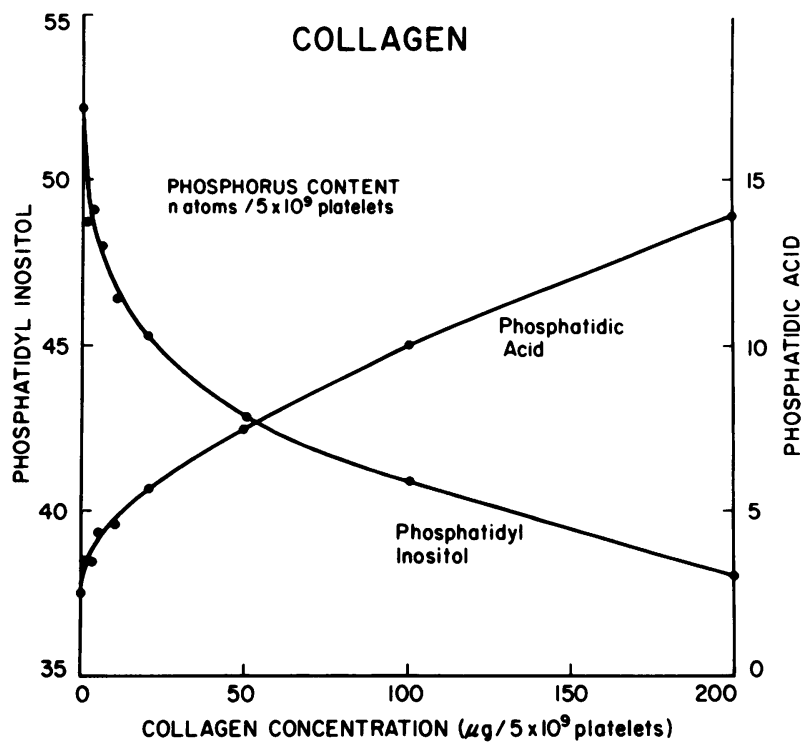

FIGURE 4 Effect of collagen concentration on the phosphatidylinositol and phosphatidic acid content of platelets. Incubations containing $5 \times 10^{9}$ platelets each were stopped $3 \mathrm{~min}$ after stimulation with the indicated amounts of collagen. Phospholipid phosphorus was quantitated as described in Methods. Small amounts of collagen generate large changes in phosphorus content of phosphatidylinositol (left ordinate) and phosphatidic acid (right ordinate).

than the gain in PA (Figs. 2 and 3; Table II). A possible explanation for this may be a series of reactions originating with accumulation of diglyceride within seconds after thrombin addition, as reported by RittenhouseSimmons (19) and subsequently by Bell et al. (18). Rittenhouse-Simmons demonstrated that this diglyceride was generated from PI by a PI-specific phospholipase C (19). A similar activity in platelet lysates has been reported by Mauco et al. (27). It was suggested that the diglyceride produced may participate in platelet secretion (19). On the other hand, Bell and associates proposed that the diglyceride is substrate for a diglyceride lipase, the presence of which they demonstrated in platelets (18). Since diglyceride derived from $\mathrm{PI}$ is very rich in arachidonic acid (10-12), combined action of PI-specific phospholipase $\mathrm{C}$ and diglyceride lipase would render arachidonate available for cyclooxygenase and lipoxygenase (18). Thus, the difference observed between loss of PI and gain in PA may be accounted for by hydrolysis of arachidonic acid from the intermediate diglyceride and its transformation into oxygenated products. This would render the diglyceride unavailable for formation of PA by diglyceride kinase (28). Bell and Majerus (29) have recently suggested that only PI is susceptible to PIspecific phospholipase $\mathrm{C}$, but not di- and triphosphoinositide.

Our observations of rapid accumulation in stimulated 


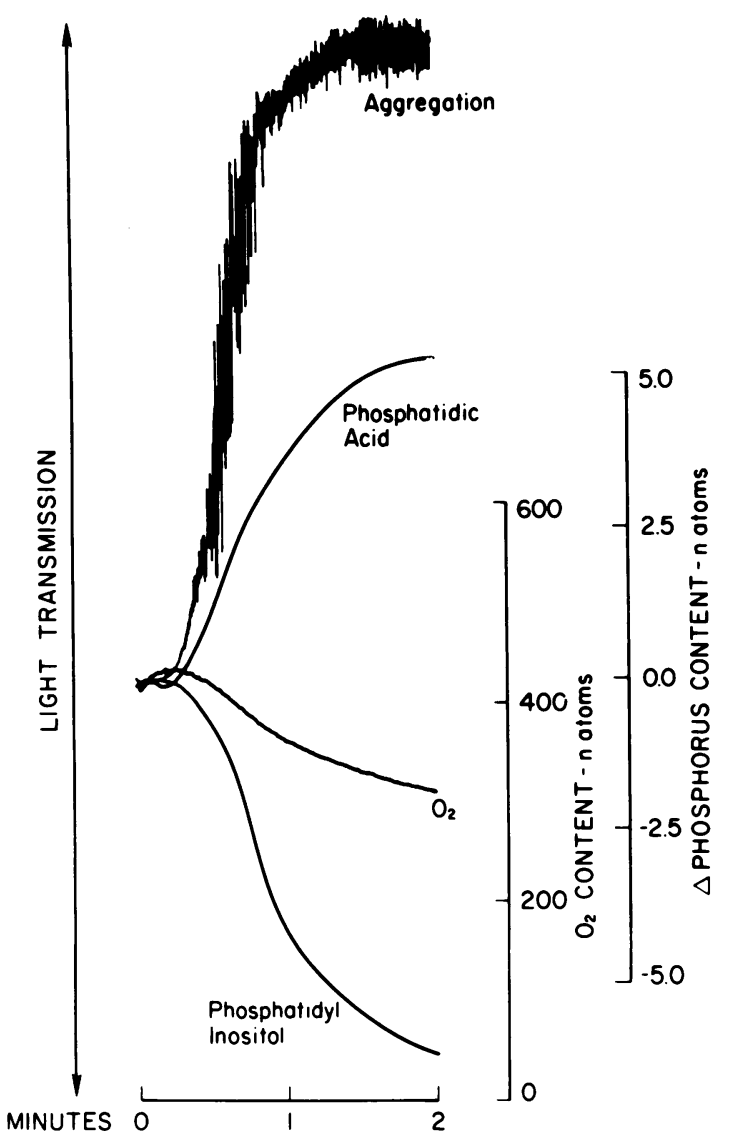

Figure 5 Time-course of concurrent changes in quantity of phosphatidic acid and phosphatidylinositol, as well as aggregation and oxygen consumption. Tracings of an aggregation curve and an oxygen consumption recording $\left(\mathrm{O}_{2}\right)$ are-shown in composite with the phosphatidic acid and phosphatidylinositol curves derived from measurements at individual time points, i.e., $5,10,15,20,25,30,40,50,60,90,120 \mathrm{~s}$. $100 \mu \mathrm{g}$ collagen was added to $5 \times 10^{9}$ platelets. After an initial small decrease in the amount of phosphatidic acid, the timecourses had similar lag phases and inflection points. (The initial rise in the $\mathrm{O}_{2}$ curve was due to the high oxygen content of the cold collagen suspension.)

platelets of LPE (Figs. 1 and 2; Tables I and II) and, to a lesser extent, of LPC, combined with an apparent decrease in PE and PC, indicate the presence of phospholipase $A_{2}$ activities distinct from those of the aforementioned PI-specific enzymes. We noted that LPE contained a smaller percentage of aldehydes (Table I) than was reported previously on the 1-position of platelet PE $(10,11,25)$. This may suggest that the LPE was generated by a phospholipase $\mathrm{A}_{2}$ acting more readily upon the diacyl form than upon the plasmalogen form of PE, a phenomenon also reported by Jesse and Cohen (30) in previous studies with platelet membrane preparations. The ethanolamine phosphoglycerides have a high content of arachidonate (10-12) and this was used as evidence favoring PE as a source of free arachidonate by these investigators (30). In contrast, Rittenhouse-Simmons et al. (31), using a different approach, suggested the plasmalogen form of $\mathrm{PE}$ as a source of arachidonate for cyclooxygenase and lipoxygenase. Partial purification of platelet phospholipase $A_{2}$ has been described (32-35). In addition, accumulation of lysophospholipids following platelet stimulation was reported in abstract form (36).

The changes in LPE and PE were completed before those in LPC and PC (Fig. 2). The changes were not due to nonspecific hydrolysis, because in unstimulated control incubations LPE or LPC did not accumulate. This difference in pattern between ethanolamine and choline phosphatides may be due to the action of phospholipase $\mathrm{A}_{2}$ activities with a diversity of specificities, or due to changes in substrate availability as a consequence of membrane rearrangement brought about by release and/or aggregation. The latter hypothesis may be more viable since the human platelet plasma membrane has been shown to possess an asymmetric phospholipid distribution, with most of the choline-containing species facing the extracellular space, and the major portion of the noncholine phospholipids on the cytoplasmic side of the membrane (37-39). Additional support for a lag in choline phosphatide metabolism is afforded by a delay in the loss of $\left[{ }^{14} \mathrm{C}\right]$ arachidonate from PC upon thrombin stimulation of prelabeled platelets (19).

Effects of inhibitors on endogenous PI-PA metabolism. Inhibition of cyclooxygenase, lipoxygenase, or thromboxane synthetase had no effect on the changes in endogenous amounts of PI and PA induced by stimulation with thrombin (Table III). This is in agreement with data recently reported by Lapetina and Cuatrecasas (4), who studied horse platelets prelabeled with $\left[{ }^{14} \mathrm{C}\right]$ arachidonate.

In contrast, alterations in availability of calcium to the platelets did affect PI-PA metabolism (Table III, Fig. 3). This is probably attributable to the divalent cation requirements of the enzymes involved. On the one hand, PI-specific phospholipase $\mathrm{C}$ is inhibited by calcium removal $(19,27)$, leading to less PI breakdown. On the other, active resynthesis is stimulated because the platelet enzymes involved in this process are magnesium-and/or manganese-dependent and are in fact inhibited by calcium $(28,40-42)$. Resynthesis of PI implies that platelet stimulation leads to operation of the classical PI-cycle (43) in its entirety. This concept is supported by the work of Lloyd et al. $(2,3)$, who showed an increase in the amount of label in PI following stimulation of platelets prelabeled with $\left.{ }^{32} \mathrm{P}\right]-$ phosphate.

Additional evidence for the importance of calcium is our observation that ionophore A23187 caused a drop in the PI content of platelets but, in comparison to thrombin, only a small increase in PA content (see 
Results). Thus, the ionophore-induced increase in $\left[\mathrm{Ca}^{2+}\right]$ may activate phospholipase $\mathrm{C}(19,27,42)$, and diglyceride lipase (18), but not diglyceride kinase (28). This indicates that calcium has a multilevel regulatory role in the metabolic fate of PI and diglyceride.

It is also likely that the concentration of ionized calcium has a regulatory function in the activation of phospholipase $\mathbf{A}_{2}$. Previous work by several investigators has emphasized the importance of calcium in the generation of free arachidonate by stimulated platelets $(15,16,44)$.

Collagen-induced alterations in endogenous PI and PA content. The time-course of changes in the level of endogenous PA showed an initial decrease $10 \mathrm{~s}$ after collagen addition. This preceded the onset of aggregation and the oxygen burst (Fig. 5). This may indicate that PA $\rightarrow$ PI metabolism occurs momentarily to a greater extent than PI $\rightarrow$ PA conversion, resulting in an initial decrease in the amount of endogenous PA (Fig. 5). Thereafter, when the quantity of endogenous PA rises and that of PI diminishes, a diglyceride lipase (18) may become activated and yield free arachidonate for cyclooxygenase, as suggested by the $\mathrm{O}_{2}$ burst (Fig. 5). However, because the loss in PI was equivalent to the gain in PA (Fig. 5), the pathway of PI-specific phospholipase $\mathrm{C}$ plus diglyceride lipase may not be the only source of free arachidonate. A phospholipase $A_{2}$ may be involved as well, since we observed an accumulation of LPE in the collagen experiments. We found that collagen stimulation leads to formation of much less thromboxane $B_{2}$ than does thrombin (unpublished observations). Thus, the relative contributions of phospholipase $\mathrm{A}_{2}$ and PI-specific pathways may be difficult to precisely quantitate.

Our data, showing a small initial decrease in the total amount of endogenous PA, are at variance with results of studies using prelabeled rabbit, human, and horse platelets $(3,4)$. In these the amount of label in PA increased before other stimulus-induced changes. This apparent discrepancy may be explained by the fact that the distribution of radioactivity in prelabeled platelets $(14,5,6)$ differs from the endogenous lipid distribution studied here.

Whether changes in quantity of endogenous lipid or of a radioactive pool with apparent high turnover are more indicative of platelet responses to stimuli cannot be answered at the present time.

In conclusion, our data indicate that dual pathways of phospholipid metabolism exist in stimulated platelets: a "true" phospholipase $A_{2}$ acting primarily on $P E$, yielding LPE as one of the products, and a pathway involving PI-specific phospholipase C, with a balance between the activities of diglyceride lipase and diglyceride kinase. Both pathways would yield free arachidonate for cyclooxygenase and lipoxygenase activities.

\section{ACKNOWLEDGMENTS}

We acknowledge the invaluable assistance of Lenore B. Safier, Harris L. Ullman, and Dr. Susan T. Silk.

This work was supported by grants from the Veterans Administration, the National Institutes of Health (HL 1882804 Specialized Center of Research, HL 07029, and 5 SO7 RR 05396 18), and the New York Heart Association.

\section{REFERENCES}

1. Michell, R. H. 1975. Inositol phospholipids and cell surface receptor function. Biochim. Biophys. Acta. 415: 81-147.

2. Lloyd, J. V., E. E. Nishizawa, and J. F. Mustard. 1973. Effect of ADP-induced shape change on incorporation of ${ }^{32} \mathrm{P}$ into platelet phosphatidic acid and mono-, di- and triphosphatidyl inositol. Br. J. Haematol. 25: 77-99.

3. Lloyd, J. V., and J. F. Mustard. 1974. Changes in ${ }^{32} \mathrm{P}$ content of phosphatidic acid and the phosphoinositides of rabbit platelets during aggregation induced by collagen or thrombin. Br. J. Haematol. 26: 243-253.

4. Lapetina, E. G., and P. Cuatrecasas. 1979. Stimulation of phosphatidic acid production in platelets precedes the formation of arachidonate and parallels the release of serotonin. Biochim. Biophys. Acta. 573: 394-402.

5. Cohen, P., M. J. Broekman, A. Verkley, J. W. W. Lisman, and A. Derksen. 1971. Quantification of human platelet inositides and the influence of ionic environment on their incorporation of orthophosphate- ${ }^{32} \mathrm{P}$. J. Clin. Invest. 50: $762-772$.

6. Leung, N. L., R. L. Kinlough-Rathbone, and J. F. Mustard. 1977. Incorporation of ${ }^{32} \mathrm{PO}_{4}$ into phospholipids of blood platelets. Br. J. Haematol. 36: 417-425.

7. Lewis, N., and P. W. Majerus. 1969. Lipid metabolism in human platelets. II. De novo phospholipid synthesis and the effect of thrombin on the pattern of synthesis. J. Clin. Invest. 48: 2114-2123.

8. Deykin, D., and D. Snyder. 1973. Effect of epinephrine on platelet lipid metabolism. J. Lab. Clin. Med. 82: 554-559.

9. Marcus, A. J. 1978. The role of lipids in platelet function: with particular reference to the arachidonic acid pathway. J. Lipid Res. 19: 793-826.

10. Marcus, A. J., H. L. Ullman, L. B. Safier, and H. S. Ballard. 1962. Platelet phosphatides. Their fatty acid and aldehyde composition and activity in different clotting systems. J. Clin. Invest. 41: 2198-2212.

11. Cohen, P., and A. Derksen. 1969. Comparison of phospholipid and fatty acid composition of human erythrocytes and platelets. Br. J. Haematol. 17: 359-371.

12. Broekman, M. J., R. I. Handin, A. Derksen, and P. Cohen. 1976. Distribution of phospholipids, fatty acids, and platelet factor 3 activity among subcellular fractions of human platelets. Blood. 47: 963-971.

13. Derksen, A., and P. Cohen. 1975. Patterns of fatty acid release from endogenous substrates by human platelet homogenates and membranes. J. Biol. Chem. 250: 9342-9347.

14. Bills, T. K., J. B. Smith, and M. J. Silver. 1977. Selective release of arachidonic acid from the phospholipids of human platelets in response to thrombin. J. Clin. Invest. 60: $1-6$.

15. Rittenhouse-Simmons, S., and D. Deykin. 1977. The mobilization of arachidonic acid in platelets exposed to thrombin or ionophore A23187. Effects of adenosine triphosphate deprivation. J. Clin. Invest. 60: 495-498.

16. Rittenhouse-Simmons, S., and D. Deykin. 1978. The acti- 
vation by $\mathrm{Ca}^{2+}$ of platelet phospholipase $\mathrm{A}_{2}$. Effects of dibutyryl cyclic adenosine monophosphate and 8- $(N, N-$ diethylamino)-octyl-3,4,5-trimethoxybenzoate. Biochim. Biophys. Acta. 543: 409-422.

17. Yoshida, N., and N. Aoki. 1978. Release of arachidonic acid from human platelets. A key role for the potentiation of platelet aggregability in normal subjects as well as in those with nephrotic syndrome. Blood. 52: 969-977.

18. Bell, R. L., D. A. Kennerly, N. Stanford, and P. W. Majerus. 1979. Diglyceride lipase: a pathway for arachidonate release from human platelets. Proc. Natl. Acad. Sci. U. S. A. 76: $3238-3241$.

19. Rittenhouse-Simmons, S. 1979. Production of diglyceride from phosphatidylinositol in activated human platelets. J. Clin. Invest. 63: 580-587.

20. Bressler, N. M., M. J. Broekman, and A. J. Marcus. 1979. Concurrent studies of oxygen consumption and aggregation in stimulated human platelets. Blood. 53: 167-178.

21. Tollefsen, D. M., J. R. Feagler, and P. W. Majerus. 1974. The binding of thrombin to the surface of human platelets. J. Biol. Chem. 249: 2646-2651.

22. Turner, J. D., and G. Rouser. 1970. Precise quantitative determination of human blood lipids by thin-layer and triethylaminoethylcellulose column chromatography. I. Erythrocyte lipids. Anal. Biochem. 38: 423-436.

23. Ryu, E. K., and M. MacCoss. 1979. Modification of the Dittmer-Lester reagent for the detection of phospholipid derivatives on thin-layer chromatograms. J. Lipid Res. 20: $561-563$.

24. Chen, P. S., Jr., T. Y. Toribara, and H. Warner. 1956. Microdetermination of phosphorus. Anal. Chem. 28: 1756-1758.

25. Marcus, A. J., H. L. Ullman, and L. B. Safier. 1969. Lipid composition of subcellular particles of human blood platelets. J. Lipid Res. 10: 108-114.

26. Hamberg, M., and B. Samuelsson. 1974. Prostaglandin endoperoxides. Novel transformations of arachidonic acid in human platelets. Proc. Natl. Acad. Sci. U. S. A. 71: 3400-3404.

27. Mauco, G., H. Chap, and L. Douste-Blazy. 1979. Characterization and properties of a phosphatidylinositol phosphodiesterase (phospholipase C) from platelet cytosol. FEBS (Fed. Eur. Biochem. Soc.) Lett. 100: 367-370.

28. Call, F. L., II, and M. Rubert. 1973. Diglyceride kinase in human platelets. J. Lipid Res. 14: 466-474.

29. Bell, R. L., and P. W. Majerus. 1980. Thrombin-induced hydrolysis of phosphatidylinositol in human platelets. J. Biol. Chem. 255: 1790-1792.

30. Jesse, R. L., and P. Cohen. 1976. Arachidonic acid release from diacyl phosphatidylethanolamine by human platelet membranes. Biochem. J. 158: 283-287.

31. Rittenhouse-Simmons, S., F. A. Russell, and D. Deykin. 1977. Mobilization of arachidonic acid in human platelets. Kinetics and $\mathrm{Ca}^{2+}$ dependency. Biochim. Biophys. Acta. 488: 370-380.

32. Apitz-Castro, R. J., M. A. Mas, M. R. Cruz, and M. K. Jain.
1979. Isolation of homogeneous phospholipase $A_{2}$ from human platelets. Biochem. Biophys. Res. Commun. 91: $63-71$.

33. Jesse, R. L., and R. C. Franson. 1979. Modulation of purified phospholipase $A_{2}$ activity from human platelets by calcium and indomethacin. Biochim. Biophys. Acta. 575: $467-470$.

34. Kannagi, R., and K. Koizumi. 1979. Effect of different physical states of phospholipid substrates on partially purified platelet phospholipase $\mathbf{A}_{2}$ activity. Biochim. Biophys. Acta. 556: 423-433.

35. McKean, M. L., J. B. Smith, and M. J. Silver. 1979. Properties of partially purified phospholipase $\mathbf{A}_{2}$ from human platelets. Proceedings of the 178th American Chemical Society National Meeting, Washington, D. C., September 1979. 62 (Abstr.).

36. Bills, T. K., M. J. Silver, and J. B. Smith. 1977. Human platelet phospholipase activities: formation of lysophospholipid in response to thrombin. Fed. Proc. 36: 1082. (Abstr.)

37. Schick, P. K., K. B. Kurica, and G. K. Chacko. 1976. Location of phosphatidylethanolamine and phosphatidylserine in the human platelet plasma membrane. J. Clin. Invest. 57: 1221-1226.

38. Chap, H. J., R. F. A. Zwaal, and L. L. M. van Deenen. 1977. Action of highly purified phospholipases on blood platelets. Evidence for an asymmetric distribution of phospholipids in the surface membrane. Biochim. Biophys. Acta. 467: 146-164.

39. Perret, B., H. J. Chap, and L. Douste-Blazy. 1979. Asymmetric distribution of arachidonic acid in the plasma membrane of human platelets. A determination using purified phospholipases and a rapid method for membrane isolation. Biochim. Biophys. Acta. 556: 434-446.

40. Call, F. L., II, and W. J. Williams. 1970. Biosynthesis of cytidine diphosphate diglyceride by human platelets. J. Clin. Invest. 49: 392-399.

41. Lucas, C. T., F. L. Call II, and W. J. Williams. 1970. The biosynthesis of phosphatidylinositol in human platelets. J. Clin. Invest. 49: 1949-1955.

42. Billah, M. M., E. G. Lapetina, and P. Cuatrecasas. 1979. Phosphatidylinositol-specific phospholipase-C of platelets: association with 1,2-diacylglycerol-kinase and inhibition by cyclic AMP. Biochem. Biophys. Res. Commun. 90: $92-98$.

43. Hokin, M. R., and L. E. Hokin. 1964. Interconversions of phosphatidylinositol and phosphatidic acid involved in the response to acetylcholine in the salt gland. In Metabolism and Physiological Significance of Lipids. R. M. C. Dawson and D. N. Rhodes, editors. John Wiley \& Sons, Inc., New York. 423-434.

44. Pickett, W. C., R. L. Jesse, and P. Cohen, 1977. Initiation of phospholipase $\mathrm{A}_{2}$ activity in human platelets by the calcium ion ionophore A23187. Biochim. Biophys. Acta. 486: $209-213$. 